\title{
Courageous Conversations in the End of Life: Implications for Critical Care Nursing
}

\author{
Therese Jamison DNP, ACNP-BC ${ }^{1}$ and Katarina Vulaj MSN, FNP-BC ${ }^{2}$
}

\begin{abstract}
:
End of Life (EOL) conversations are complex entities that effect individuals, families, surrogates, and care providers across the lifespan as well as across the care continuum. The sensitive nature of these courageous conversations can be difficult for all parties involved. In this review of the literature (ROL), best practice and clinical practice protocols instituted within the last five years will be identified in an effort to provide critical care nurses and advanced practice nurses with the current practice guidelines. Both qualitative and quantitative studies will be identified. Definitions of EOL including a good and dignified death will be explored. Challenges surrounding EOL conversations as well as facilitators for providing EOL care including educational resources will be highlighted. Interprofessional collaboration is essential for providing care that is guided in humanism as well as evidenced based care.

KeyWords: End of Life; Palliative Care; Critical Care Nursing; Advanced Practice Nursing; End of Life Care in the Critical Care; Advanced Directives (AD)
\end{abstract}

\section{INTRODUCTION}

An understanding of the natural progression of life amongst the general population seems to be out of focus. As new progress is made with medical advances, so does the expectation that people will be cured of their ailments and live longer. But what happens when their disease processes advances beyond treatment? How can we help patients and loved ones bring into focus a natural and peaceful death? The importance of these questions becomes paramount when one in five patients expires shortly following an intensive care unit (ICU) admission (Chow, 2014). Grant et al. (2013) forecast by 2030, 20\% of the population in America will be 65 years or older with the fastest growing population being folks 85 years and older. Unfortunately,during a terminal illness, $40 \%$ of Medicare patients who expire will do so while in the ICU. During the last year of a patient's life, $25 \%$ of all Medicare spending is incurred. Additionally, less than 5\% of patients who are terminally ill overcome Cardio Pulmonary Resuscitation (CPR) to eventually obtain discharge from the hospital, instead they could require mechanical ventilator support for an unspecified amount of time; therefore, significantly impeding the patient's quality of life (Scott, 2010). An essential part of the nurse's duty is to give bad news and provide emotional care to patients and their family (Kurian et al, 2014). Eventual systemic organ failure seen with terminally ill patients may lead to the use of the following medical interventions: mechanical ventilation, artificial feeding (via nasogastric (NG) tube, cortrak or peg tube), hemodialysis, chemotherapy/radiation, surgery, blood transfusions, antibiotics and admission to the ICU (Scott, 2010).

Courageous conversations in End of Life (EOL) care remain complex entities for advanced practice registered nurses (APRN) and critical care nurses (CCN) in the intensive care units. According to the End-of-Life Nursing Education Consortium (ELNEC) (2012), executive summary, nurses spend more time than any other healthcare professional in providing EOL care. "The nurse must be the advocate for the patient and family, the constant in a sea of numerous caregivers (ELNEC, 2012, p. 14).Nurses are expected to take on the responsibility to engage the patient and the patient's family in discussions regarding EOL wishes (Ryan and Jezewski, 2012). This time is filled with diverse and intense emotions for patients, families, surrogates, and healthcare providers. The aim of this review of the literature will be to explore the current standards, clinical practice guidelines, best practice, as well as evidenced based practice for EOL with a focus on nursing implications.

\section{EOL AND RELATED DEFINITIONS}

As defined by the Institute of Medicine (2015), EOL care refers "to the processes of addressing the medical, social, emotional, and spiritual needs of people who are nearing the end of life. It may include a range of medical and social

${ }^{1}$ Corresponding Author: Tjamison@madonna.edu 
services, including disease-specific interventions as well as palliative and hospice care for those with advanced serious conditions who are near the end of life" (p.27). The American Nurses Association (2010), position statement on EOL, contest that nurses have an obligation to provide comprehensive and compassionate support for patient, families, and surrogates.

Nurses, as well as interprofessional team members, need to assist patients and families with the varied terminologies associated with EOL. The term "do not resuscitate" can have ambiguous meanings (everything done, lifesaving, life sustaining therapies (LST), no-code) unless clear explanations are provided (Browning, 2011; Wiegand \& Grant, 2014). Furthermore, as described byWiegand and Grant (2014), "withholding LST involves deciding not to initiate treatment with the understanding that the patient will probably die without the intervention, whereas withdrawal of LST involves deciding to stop or remove treatment with the knowledge that the patient will die following the change in therapy" (p.62).There is no universally agreed upon definition of medical futility (Chow, 2014).

The premise of a "dignified" death and a "good" death is closely linked to EOL.Bratcher (2010) conducted a qualitative exploratory study of $15 \mathrm{RNs}$ working in a 12 bed ICU in an attempt to define a good death. Eight main themes emerged from data analysis, with three themes mentioned most often: the patient does not die alone, the patient does not suffer, and acceptance of death by the patient and loved ones. In a qualitative study, conducted by Volker and Limerick (2007), 19 oncology APRNs interviewed patients in EOL. The interviews were audiotaped and analyzed utilizing a phenomenological approach. The results formulated three themes for a dignified death: going in peace (connecting with loved ones, conveying final messages, dying in a nonchaotic environment) maintaining body integrity (bodily functions, appearance, and privacy), and dying on their own terms (respect for personal preferences).

\section{Specific Challenges for Nurses Providing EOL CARE}

There can be a lack of understanding on behalf of patients and their families that medical intervention may solely protract the course of death while many times introducing upsetting symptoms and consequences, which after the passing of the patient bring on feelings of remorse (Searl, 2015). Presence of struggle with end of life decisions is frequent in the ICU with family members expressing difficulty with accepting and putting in action a patient's wishes regarding care. Additionally, they may be unfamiliar with the treatment available, or they may experience overall discrepancy among themselves regarding the next course of action (Scott, 2010). Second to this lack of understanding on medical interventions with potential feelings of remorse with EOL decisions, the utilization of Advanced Directives (AD) becomes extremely important.ADs are written documents that express the extent of intervention from healthcare providers. In a systematic synthesis of reported results from three survey studies conducted on critical care, emergency department, and oncology nurses, Ryan and Jezewski (2012) identified that the level of knowledge on ADs was $60 \%$ correct and that nurses reported a moderate level of confidence related to AD. As discussed by Nelson and Nelson (2014) barriers to carrying out advanced directives exist on multiple levels including: patient, surrogate, provider, and health system. Barriers at the patient level include: loss of mental stability such as dementia (the patient would not be able to make the decision on their own or be reliable), not finishing an $\mathrm{AD}$, no actual decision regarding care for oneself, $\mathrm{AD}$ not easily available in time of need to family or provider, change of mind regarding wishes placed in the $\mathrm{AD}$, and lack of thorough thought regarding one's wishes before completing this document. At the surrogate level obstacles are as follows: disagreement regarding standards and principles of care between patient and surrogate; emotional and situational strain due to significance of decision to be made by surrogate regarding patient care; presentation of unexpected situations not discussed previously by patient and surrogate, therefore not in the AD. Providers may have barriers including: insufficient knowledge regarding putting together an $\mathrm{AD}$, not sufficient reimbursement and time to have conversations regarding $\mathrm{AD}$, fear of liability/litigation, inability to predict disease course for patient, and not having similar beliefs regarding EOL care. General health system barriers regarding carrying out an AD are: lack of or insufficient communication between primary care physician and hospital, AD is not automatically adaptable to inpatient medical orders, and dialogue regarding $\mathrm{AD}$ in an acute care setting being not reimbursable (Nelson \& Nelson, 2014).

\section{ENVIRONMENT}

A work environment can be an inhibiting factor for nurses in being effective patient advocates (Wiegand \& Grant, 2014). There is a high possibility of exhaustion for nurses who provide direct care for terminally ill patients. Additional stressors include: lack of appropriate staffing, coworker conflict, heavy workload, emotionally arduous patients and families, along with prolonged exposure to dying and death (Chow, 2014). In a review of the literature of 13 quantitative and 9 qualitative studies, Espinosa, Young and Walsh(2008), identified barriers to ICU nurses in 
providing terminal care: nurses may face a lack of consensus between the interdisciplinary team regarding prognosis of patient; insufficient pain management for patient; naïve/ungrounded beliefs of patient's family regarding patient prognosis; the inability of the nurse to deal with the situation at hand; a deficiency in the nurse's education and/or experience; and hindering work environment including the layout and size, and the noise of unit.

\section{COMMUNiCATION}

Quality communication with family members regarding a patient's clinical trajectory is essential to formulating treatment decisions. According to Scott (2010), nurses are first in line when families are seeking guidance regarding patient EOL care.Throughout the literature reviewed, the one common theme noted was the need for communication with patient and family. Barriers to this communication on behalf of the family included: lack of comprehension, overall displeasure, unachieved goals of care, tension or dispute with providers and therefore a decreased element of trust, enhanced feelings of obligation and remorse, and not being prepared to have such a conversation (Kalowes, 2015). In a survey performed by Malloy, Virani, Kelly and Munevar (2010) on 333 participants who attended an ELNEC conference, the authors identified several key areas for improvement in communication: discussion of bad news, talking with physicians regarding palliative care issues, discussing spiritual concerns, and talking with patients ands families from different cultures. Consistently, nurses with less experience (less than 10 years) had more difficulty in these areas. According to Searl (2015), there may also be a lack of inclination to discuss the patient's prognosis by the provider. This can be due to the presence of conflicting sentiments on prognosis or lack of selfassurance and/or experience in discussing a poor prognosis. Furthermore, Kalowes (2015) contended additional barriers to quality communication including: the provider along with the nurse not willing to take away optimism from the patient or patient's family; nurse's uneasiness with dying and EOL care; patient or family refusal to accept patient prognosis or consideration or palliative/hospice care; families obtaining information on their own accord not directly from providers, but outside resources, or partial members of the team therefore getting an incomplete prognosis. In a post-test survey design using a convenience sample of ICU nurses, Kurian et al, (2014) identified that $46 \%$ of the nurses indicated that they had insufficient education, training, and experience with bereavement care.As identified by Scott (2010), nurses voicedthe desire to have been instructed, while in nursing school, on how to carry out conversations with patients and their family regarding EOL.

\section{FAMILY AND PATIENT DYNAMICS}

According to Browning (2010), family indecisiveness regarding EOL care may be associated with: lack of comprehension about consequences of life support, insufficient data regarding patient illness trajectory, and refusing to recognize poor prognosis. Hinkle, Bosslet and Torke (2015) conducted a systematic review of literature regarding EOL care in the ICU and influences on family satisfaction. The reviewers noted in one study that half of the family members had no desire to be involved with the decision-making process regarding EOL care. Apart from this, there may be instances where the family is not sure of what the right course of action should be for their loved one. The family is under an extremely stressful circumstance when a loved one is ill in the ICU. This stress is magnified when the patient's condition changes quickly further amplifying the uncertainties regarding the patient's outcome. Greater satisfaction in the decision making process occurred with the presence of a higher education level of the patient and family as well as a reached consensus of stopping medical intervention between physicians. Overall satisfaction considerably increased with EOL care when interaction and communication between the patient/family and providers occurred (Hinkle, Bosslet \&Torke, 2015).

\section{Facilitators in Providing EOL Care}

In a review of the literature conducted by Searl (2015), 10-15\% of ICU's utilize mortality-predicting metrics. The use of these metrics to confer course of palliative or EOL care may: improve family satisfaction with dying process in hospital, provide extra time for families to understand the course of the patient's prognosis, reduce staff stress who are tending to a patient with an inevitable/looming death, and ameliorate the value of EOL care (Searl, 2015). Three common scoring systems used by the ICU to obtain a severity of illness score include: The Acute Physiology and Chronic Health Evaluation IV (APACHE IV) system, Mortality Probability Admission Model-III (MPM-III), and The Simplified Acute Physiology Score 3 (SAPS 3) (Kalowes, 2015). Considerable patient data is collected and used with each scoring system to compile a numerical value that can assist providers to determine possible interventions. All three of these predictive instruments provide a useful severity of illness score to guide clinical care. "Empowered by knowledge related to end-stage disease criteria, performance status, prognostic (severity of illness score and mortality) information, and patient preferences, providers will be prepared to serve as information brokers and advocate effectively for patients and families in collaboration with the interprofessional team" 
(Kalowes, 2015, p. 160).

Nelson et al. (2011) identified nurses as having a leading role when integrating palliative care in the ICU. The authors provided practical suggestions for $\mathrm{CCN}$ : take a leadership role in interdisciplinary team meetings to identify and implement quality improvement measures for palliative care by supporting teamwork, as well as creating a culture that supports palliative care; identify obstacles for palliative care and how to overcome these; participation of $\mathrm{CCN}$ in family meetings; support palliative care education; as well as mechanism to evaluate these quality improvement initiatives. Additionally, Brennan, Prince-Paul and Wiencek (2011) described other suggestions: maximize symptom management, according to patient and family'spersonal/cultural preferences; educate patient and family on what to expect in the final hours; assess for rituals to attend to the patient's personal preferences; encourage family members for self care, such as breaks for sleep and nutrition; create a "get to know you" poster in the patient's room so providers can understand the patient's life; encourage personal items (such as pictures, blankets, etc); encourage the family to be involved in care.

Hinkle, Bosslet and Torke (2015) described a single center, before and after study, where family members were purposefully included in ICU rounds. The intervention resulted in a significant improvement in satisfaction with communication and decision making support for families of survivors. Additionally, these same authors reported a randomized control trial that assessed the effectiveness of written information to prepare families members for the dying process after withdrawal of life saving interventions. The families receiving the written information reported greater satisfaction than those not receiving the information.

Espinosa, Young, Symes, Haile and Walsh (2010) conducted a descriptive phenomenological research study. Purposeful sampling was utilized to select participants who varied in gender, age, and nursing experience. The sample consisted of $18 \mathrm{CCN}$. One of the aims of the study was to identify coping strategies. Those identifiedwere considered mostly positive and adaptive. These strategies included: building trust with the family (once the nurse builds trust, the family relies on the nurse for information); crying (nurses did not feel that crying interfered with the care delivered, but rather helped the family cope with the loss); humor (was used to take the stress off the situation); talking to others about terminal care (conversations with other nurses can provide reassurance and support); and avoiding care for the terminal patient (nurses noted that some nurses are better in providing terminal care, others refrained from providing care to the terminally ill second to situations in their own personal lives).

Ina descriptive study conducted on 50 nursing students using high fidelity simulators dealing with dying critically ill patients/families, the use of The Palliative Care of Dying Critically Ill Patients Algorithm, along with case review/discussion, raised EOL Nursing Education Consortium Knowledge Assessment Test scores and self confidence among student nurses regarding end of life care (Grossman, 2013). The components of the algorithm included: identify if the patient had an $\mathrm{AD}$ and ensure that the $\mathrm{AD}$ is sustained/updated by the patient/family; establish the decision maker for the patient's care; consult with the palliative care team; assign the same nurse (s) to the patient to maintain a therapeutic relationship; collect all possible data regarding the patient/family's desire and needs; communicate frequently, hourly or more so, with family/significant others; allow flexibility with visiting hours; include family if desired in providing care; assist in setting up a patient/family/ICU meeting; set up a plan for assisting with the patient's desires/needs for remaining hours of life, including a transfer out of the ICU; communicate with the providers in the transition out of the ICU (Grossman, 2013).

\section{EDUCATIONAL RESOURCES FOR EOL CARE}

To the validate and promote the education of the APRN and CCN, The American Association of Critical Care Nurses, Hospice and Palliative Nurses Association, the National Association of Hospice \& Palliative Care,The Center to Advance Palliative Care, the National Palliative Care Research Center, and ELNEC all provide continued support for palliative and EOL care. ELNEC (2012) is a national education program designed to enhance palliative care in nursing. The City of Hope and the American Association of Colleges of Nursing administer the training. The Robert Wood Johnson Foundation originally funded the program in 2000. In 2003, the project has received additional support from many other funding sources. ELNEC-Core's nine original modules contain information on: nursing care at the end of of life, pain management, symptom management, ethical/legal issues, cultural/spiritual considerations, communication, grief/loss/bereavement, achieving quality care, and care at the time of death.In 2006, ELNEC debuted ELNEC-Critical Care, funded by the Archstone Foundation. This course is written and designed by nurses for nurses in the following areas: undergraduate and graduate nursing faculty who educate critical care nurses, critical care CE providers, hospice nurses who provide consults in critical care settings, as well as APRN and nurses working in critical care settings (ELNEC, 2012).Malloy, Thrane, Winston, Virani, and Kelly (2013) surveyed nurses following one of the nine ELNEC courses between April 2009 and May of 2010. For this 


\section{American Research Journal of Nursing, Volume 1, Issue 3, 2015}

ISSN 2379-2922

study, 605 nurses, practicing in diverse healthcare settings, responded to a survey about self-care that was developed by the ELNEC investigators and faculty. Those self care strategies with the highest means were: taking at least a one week vacation per year, having meaningful relationships outside of work; and having meaningful relationships at work.

\section{CONClusions}

Nursing continues to be the integral thread in the weave of courageous conversations that occur in critical care settings for EOL. Multidimensional factors can either impede or enhance these EOL conversations. One can either choose to ignore the research and best practice as identified in this relevant literature review, or empower oneself as well as the interprofessional team to provide care that is delivered with high quality, integrity and humanism. One would hope healthcare providers make the latter choice so that patients, families, and surrogates can experience a natural and peaceful death.

\section{REFERENCES}

[1] American Nurse Association. (2010). Registered nurses roles and responsibilities in providing expert care and counseling at the end of life: Position Statement. Retrieved from: www.nursingworld.org/...position-statements/etpain14426.pdf

[2] Bratcher, J.R. (2010). How do critical care nurses define a "good death" in the intensive care unit? Critical Care Nursing Quarterly, 33(1), 87-99.

[3] Brennan, C.W., Prince-Paul, M., \& Wiencek, C.A. (2011). Providing a "Good Death" for oncology patients during the final hours of life in the intensive care unit. AACN Advanced Critical Care, 22(4), 379-396.doi: 10.1097/NCI.0b013e31823100dc

[4] Browning, A.M. (2011). End-of-life care in the ICU: Helping families decide. Nursing2011Critical Care, 6(1), 5-8.

[5] Chow, K. (2014). Ethical dilemmas in the intensive care unit: Treating pain and symptoms in noncommunicative patients in the end of life. Journal of Hospice \& Palliative Nursing, 16(5), 256-260.doi: 10.1097/NJH.0000000000000069

[6] End-of-Life Nursing Education Consortium (ELNEC) (2012). History, statewide effort and recommendations for the future: Advanced palliative nursing care. Retrieved from: www.aacn.edu/elnec/publications/ELNEC-Monograph.pdf.

[7] Espinosa, L., Young, A., \& Walsh. T.(2008). Barriers to intensive care unit nurses providing terminal care: An integrated literature review. Critical Care Nurse Quarterly, 31(1), 83-93.

[8] Espinosa, L., Young, A., Symes, L., Haile, B., \& Walsh, T. (2010). ICU nurses' experiences in providing terminal care. Critical Care Nursing Quartely, 33(3), 273-281.

[9] Grant, M., Wiencek, C., Virani, R., Uman, G., Munevar, C., Malloy, P., \& Ferrell, B. (2013). End of life care education in acute and critical care: The California ELNEC project. AACN Advanced Critical Care, 24(2), 121-129.doi: 0.1097/NCI.0b013e3182832a94

[10] Grossman, S. (2013).Development of the palliative care of dying critically ill patients algorithm: Implications for critical care nurses. Journal of Hospice \& Palliative Nursing, 15(6), 355-359.doi: 10.1097/NJH.0b013e3182987b1c

[11] Hinkle, L.J., Bosslet, G.T., \&Torke, A.M.(2015). Factors associated with family satisfaction with end-of-life care in the ICU. CHEST, 147(1), 82-93. doi: 10.1378/chest.14-1098

[12] IOM (Institute of Medicine). 2015. Dying in America: Improving quality and honoring individual preferences near the end of life. Washington, DC: The National Academies Press.

[13] Kalowes, P. (2015). Improving end-of-life care prognostic discussions: Role of Advanced Practice Nurses. $A A C N$ Advanced Critical Care, 26(2), 151-166.doi: 0.1097/NCI.0000000000000086

[14] Kurian, M.J., Sulata, D., James, A., James, C., Joseph, L., Malecha, A.T.,...Mick, J.M. (2014). Intensive care registered nurses' role in bereavement support.Journal of Hospice and Palliative Care Nursing, 16(1), 3139.doi:10.1097/NJH.0000000000000018

[15] Malloy, P., Thrane, S., Winston, T., Virani, R., \& Kelly, K. (2013). Do nurses who care for patients in palliative and endof-life settings perform good self-care? Journal of Hospice \& Palliative care, 15(2), 99-106.doi: 10.1097/NJH.0b013e31826bef72

[16] Malloy, P., Virani, R., Kelly, K., \& Munevar, C. (2010). Communication skills of nurse in palliative care.Journal of Hospice \&Palliative Care, 12(3), 166-174.

[17] Nelson, J.E., Cortez, T.B., Curtis, J.R., Lustbader, D.R., Mosenthal, A.C., Mulkerin, C.,..Puntillo, K. A. (2011). Integrating palliative care in the ICU: The nurse in a leading role. Journal of Hospice \& Palliative Care, 13(2), 89-94.doi: 10.1097/NJH.0b013e318203d9ff

[18] Nelson, J.M, \& Nelson, T.C.(2014). Advanced directives: Empowering patients at the end of life. The Nurse Practitioner, 39(11), 35-40.doi: 10.1097/01.NPR.0000454979.98327.89

[19] Ryan, D.,\& Jezewski, M.A. (2012). Knowledge, attitudes, experiences, and confidence of nurses in completing advanced 
American Research Journal of Nursing, Volume 1, Issue 3, 2015

ISSN 2379-2922

directives: A systemic synthesis of three studies. The Journal of Nursing Research, 20(2), 131140.doi:10.1097/jnr.0b013e318256095f

[20] Scott. S.A. (2010). Life-Support Interventions at the end of life: Unintended consequences. American Journal of Nursing, $110(1), 33-39$.

[21] Searl, M.F. (2015). A case for the use of validated physiological mortality metrics to guide early family interventions in intensive care unit patients. AACN Advanced Critical Care, 26(1), 13-22.doi: 10.1097/NCI.0000000000000063

[22] Wiegand, D.L., \& Grant, M.S. (2014). Bioethical issues related to limiting life-sustaining therapies in the intensive care unit. Journal of Hospice \& Palliative Nursing, 16(2), 60-64.doi: 10.1097/NJH.0000000000000049

[23] Volker, D.L., \& Limerick, M. (2007). What constitutes a dignified death? The voice of oncology advanced practice nurses. Clinical Nurse Specialist, 21(5), 241-247. 